\title{
CONCLUSION
}

\section{The Intricacies of Transnational Relationships in the Promotion of Gender Equality and Women's Empowerment}

Nnenna Okoli

\section{Introduction}

This special issue examined the exchange of knowledge and experiences and the relationships built in transnational spaces between international development volunteers (IDVs) and partner organizations (or volunteer receiving organizations) in the Global South. The focus of analysis running throughout this collection was an examination of the work of transnational actors in gender equality and women's empowerment (GEWE) programming.

The papers drew from a database of qualitative research conducted in ten different countries (Ghana, Kenya, Malawi, Tanzania, Senegal, Ghana, Nepal, Vietnam, Guatemala and Peru) by emergent and prominent researchers from the Global North and South between 2018 and 2020. The overall research design was collaborative, participatory and reciprocal, in that the knowledge and perspectives of Global South partner organizations were solicited from the initial planning stages to the final execution of the research. The research project also prioritized the development of budding scholars' research capacity through their participation in the creation of the research design, data collection, data analysis and the presentation of the findings. In this way, this special issue shifts the spotlight from the agency of actors in the Global North's to a (re)valuing of the voices and contributions of people in the Global South. It recognizes the value of mutual learning facilitated through international volunteerism programs to realize SDGs like gender equality.

The contributions in this collection drew from multiple theorical perspectives that share common analytical perspectives of women in development (WID), women and development (WAD), and gender and development (GAD). In their analyses of gender equality, the discrete papers employed discursive normative theory, identity-based theories, feminist theories, critical development theory, and the human capabilities framework. Drawing on these diverse but connected theories to explain qualitative research findings, contributors to this collection confirmed 
the significant multi-dimensional contributions that IDVs offer to realize GEWE targets in the Global South. Importantly, IDVs make these contributions through collaborative and generative interactions with partner organizations in line with transnational feminist values that eschew strict economic development in celebration of human and relational interactions.

\section{Core Themes}

The relational dimension of the transnational interactions between IDVs and partner organizations in the Global South fills an important gap in the scholarship, moving beyond a study of the experiences and impacts of international volunteers. Unlike most quantitative research on this subject, the collection's qualitative research methodology enabled the researchers to unpack the subjective meanings that partner organizations ascribed to processes, actions, interactions, and relationships with volunteers as they relate to GEWE programming. These interactions extended beyond the technical and "functional" revealing a deliberate cultivation of human relationships based on trust, inspiration and friendship in both formal and informal spaces. These interpersonal interactions greatly strengthened IDVs' contributions to gender equality outcomes.

Various case studies in this collection illustrated how IDVs' interpersonal relationships influenced their pursuit of gender equality targets. For instance, the relational complementarities of IDVs facilitated the acknowledgement and acceptance of alternative notions of womanhood and gender equality in the host communities while simultaneously informing the IDVs of cultural specificity and local context. The case studies also highlighted that the informal encounters and the exchange of personal intimate knowledge between IDVs and host communities greatly catalyzed the positive shift in local women's and men's perceptions of their rights and assigned gender roles.

On the other hand, these case studies also highlighted challenges that emerged from these interpersonal relationships across different cultural and geographic contexts. In cases where potential relationships of trust and respect were jeopardized by factors like IDVs' disregard for local culture and behavioral norms, communities were justifiably hostile and unreceptive of IDVs. As these case studies imply, volunteers with low levels of intercultural competence, empathy and recognition of cultural relativism consequently diminished whatever impact they could have made in promoting gender equality and empowerment in the communities where they served.

Other important insights offered by the articles in this special issue include the strength and value of Global South actors; the primacy of their agency; and the critical importance of their voice in their relations and interactions with IDVs, donor 
organizations, and other Global North partners. Evidence from this research highlights that the many benefits of these transnational relationships can be fully reciprocal, including contributions such as capacity building, knowledge sharing, intercultural learning, and innovation. They accentuate that Global South organizations are not simply recipients of funding, technical assistance, and the vessels of resources from the Global North; but they are also valuable contributors to the development of IDVs' skills and capacities.

Partner organization staff expressly underscored the advantages of hosting volunteers whose expertise and distinctive values on gender relations enhanced organizational perspectives and actions in pursuit of GEWE. They celebrated the genuine value of these relationships that were felt as mutually symbiotic and collaborative. This was particularly true in cases where both parties engaged in a partnership that upheld local organizational and communal principles and priorities - especially when local customs clashed with Western feminist ideals.

The findings also speak to literature on the intersections between gender mainstreaming, volunteerism, and international development. Gender has been mainstreamed to varying degrees in development organizations' structures, systems, processes, policies, and programs. However, its interpretation and implementation by development actors have varied and mostly produced disappointing outcomes. Using the Vietnamese case study, Lan Nguyen speaks to extant critical feminist criticisms of gender mainstreaming in development policy and practice. Partner organizations in this context either downplayed the important role of civil society in pushing the GEWE agenda or adopted a myopic conceptualization of gender equality. Central to this thesis was the neglect of the relational and structural dimensions of GEWE, and a focus on its instrumental and depoliticized form. In Vietnam, the existence of national pro-GEWE policies and the significant representation of women in the public sphere were erroneously equated with the realization of gender equality and empowerment. This superficial approach can be attributed to partner organizations' attempt to display some form of gender-sensitivity in their projects to strategically position themselves for continued receipt of donor funding. To the chagrin of critical feminists, this same approach tends to dominate development practice in the Global North.

Characteristic of mainstream development's conceptualization of empowerment, the version of empowerment described in this collection was often bereft of political elements necessary to transform oppressive gendered power relations and structures. For instance, Saint-Denis' analysis points to the subjugation of the inherently political project of empowerment to the neoliberal economic agenda through a narrow focus on assets and skills transfer to expand women's economic opportunities. Not to be mistaken, however, IDVs were able to harness their formal and informal interactions with beneficiaries of GEWE programs to encourage positive 
attitudinal and behavioural changes within communities, while also securing economic benefits for individual women.

Likewise, the paper by Tiessen, Laursen, Lough (with Mirza) elaborates on IDVs' contribution towards transformative social innovation and economic empowerment for women. This paper also addressed women's social vulnerabilities that are rarely covered in the literature, such as time poverty. In Tanzania and Ghana, IDVs recognized the unequal burden of unpaid domestic and care work on women's poverty due to its constraints on their opportunities for paid employment, skills training and education (UN Women, 2019). Hence, IDVs began to address these structural limitations by introducing equality-promoting programs such as child-care services during trainings and workshops to enable women to participate in GEWE programs.

IDVs further promoted women's political empowerment and collective mobilization by offering mentorship, motivation, and entrepreneurial and leadership training to women in the communities, as well as technical, research and advocacy support to partner organizations for political transformation. As Mpogazi emphasized throughout her paper, the efforts of IDVs working in partnership with Global South organizations helped to amplify women's political voice, capacities, representation, and collective participation. This was particularly evident in the case description of the network of councillors working in the district of Niayes (RCAN) in Senegal. Each of these activities served to enhance the visibility of the gender equality cause, buttressed the agenda for women's empowerment, and emphasized the critical importance of GEWE activities to policymakers.

In spite of the many contributions that IDVs make to GEWE programs, these improvements are often clouded by inter-cultural tensions between IDVs, their host organizations, and communities. Mirroring post-colonial feminists' critiques of Eurocentric feminisms, findings from Peru, Ghana and Kenya all highlight the tendency of IDVs to universalize and homogenize the oppression of women across racial and cultural categories while simultaneously essentializing "Third World women" as victims of their oppressive patriarchal cultures. Communities' resistance to IDVs' foreign ideas on ideal gender relations were prevalent in most of the countries represented in this collection. At the same time, Sadat's findings suggest a potentially different outcome resulting from these intercultural differences. This paper found that IDVs may be strategically positioned to influence gender norms and stereotypes because communities may be more receptive to gender equality messaging and advocacy coming from external agents like IDVs.

Points of cultural friction were particularly evident in cases where female IDVs contravened their host communities' traditional gender norms pertaining to women's responsibilities and mode of conduct. Interestingly, such tensions were far 
less observable when male IDVs pushed back on traditional gender roles and stereotypes. As Shahadu Bitamsimli highlighted in his paper, partner organizations painted men as strategic allies in reforming patriarchal gender norms to ensure the success of GEWE initiatives. The cross-cultural interactions and exchanges between male IDVs and their host communities created space for imagining alternative forms of gender division of labour in situations where unpaid care and domestic work were more evenly distributed between women and men. By embodying unconventional masculinities that transcend patriarchal cultural norms, male IDVs served as models for male community members to advance the goal of gender equality and empowerment. Considering men's general resistance to gender mainstreaming policies and programs (see Parpart, 2014), men's reorientation and inclusion in GEWE programming is beyond integral to the success and sustainability of these programs. Getting buy-in from men to advance and advocate for GEWE programming effectively mitigates resistance and resentment among men that would otherwise be excluded.

Another important highlight of the papers in this collection is the exertion of agency by Global South partner organizations working with IDVs. Previous literature already acknowledges that IDVs are instrumental in building the capacities of partner organizations through the transfer of skills, knowledge, and resources essential to the successful implementation of GEWE programs. However, Sadat provides a new perspective by emphasizing the reciprocal nature of capacity building and capability development; such that benefits accrue to both IDVs and partner organizations. In this way, asymmetrical power relations are evaded. Through reciprocity and partnership-based relationships, partner organizations were able to provide localized and contextualized knowledge on gender issues while correspondingly enjoying increased organizational efficiency and innovative ideas as a result of their collaborations with IDVs. As Rouhani illustrates, the transnational interactions between IDVs and partner organizations facilitate the creation of novel ideas and enhance the latter's strategies to dismantle structural barriers to women's rights through the synthesis of cross-cultural ideas on gender equality. Hence, the transnational space in which IDVs and partner organizations operate is characterized by a multi-dimensional flow of ideas, resources and skills that mutually enhances the cosmopolitan identities of both parties and flattens power differentials. Within this space of transnational interaction and reciprocity, Global South organizations were able to exercise their agency. Findings buttress the conclusion that their locally informed voices on gender issues were respected by IDVs as valuable and insightful.

The agency of Global South organizations was also evident as they navigated the structural inequalities stemming from overreliance on donor funding for their operations. Laursen delved into the negotiation, compromise, resistance, and 
decision-making processes of these organizations in relations to the compatibility of donors' feminist objectives with their organizational values, interests, and priorities. While Feminist Foreign Policies have decisively altered donor-recipient relationships by intensifying the (sometimes unfavorable) GEWE conditionalities attached to aid, Global South organizations have employed various tactics to assert their autonomy where possible. The organizations' preservation of their own ideologies, values, and objectives at the expense of donor funds reflects their preference for greater autonomy and ownership of their programs. Findings from this paper suggest that these choices were largely determined by donor requirements and expectations; donors sought to fund potential recipients based on good fit and compatibly with their cultural norms, regardless of their innate capabilities. These findings further highlight the importance of cultural awareness and sensitivity on the part of donors and IDVs in their relations with partner organizations in the Global South

\section{Implications}

Taken together, the papers in this collection contribute to our knowledge on the unique roles of IDVs and Global South partner organizations as transnational actors in international development. The discrete papers each pay attention to the intricacies of the transnational relationships between IDVs and partner organizations. By analyzing these intricacies, they consequently uncover a form of power relations that transcend dominant conceptions of international development volunteering. In terms of capacity building for example, the collection shows that capacity development is jointly shaped by both IDVs and partner organizations; such that there is a mutual and enriching exchange of skills and knowledge between both actors.

The analyses also recognize partner organizations as active transnational actors with distinct experiences, perceptions, and expectations. These partners are often assertive in planning and implementing GEWE programming to affect gender equality outcomes. Although the existing literature has mainly privileged Northern organizations' judgements of the impact of international volunteerism, this collection offers more inclusive insights into the experiences of partner organizations. It demonstrates that partners are not merely beneficiaries but are active contributors to transnational spaces.

This process brings a more dynamic lens to our understanding of the interpersonal relationships between IDVs and partner organizations that are pivotal to understanding the extent of IDVs' contributions to the processes and outcomes of the GEWE programming. This lens also highlights the distinctive contributions of international development volunteering as a model of transnational relations to the diffusion of norms, ideas, information, skills, and principles geared towards the transformation of gender relations and structures in the Global South. Although 
the specific contexts differ, the collection provides many detailed accounts of the dynamics of GEWE programming in the Global South to illustrate these points across diverse communities. These accounts illustrate important messages such as the negotiation of power and interests between Southern partner organizations, Northern donors, and IDVs; the cultural tensions inherent in the implementation of GEWE programs; the setbacks and successes in achieving transformation in gender relations; the process of engaging with boys and men in pursuit of GEWE; and the prominent role IDVs can potentially play in all of these processes.

\section{Recommendations}

The nuanced complexity, merits, and limitations of the transnational interactions and relationships between IDVs and partner organizations have been illustrated well throughout the papers. From the evidence presented, several recommendations emerged to enhance the impact of IDVs in facilitating GEWE programming and development outcomes broadly.

Perhaps the most glaring concern articulated by partner organization staff was the cultural friction that characterized IDVs' promotion of gender equality. The papers point to tensions surrounding IDVs' imposition of feminist standards that contravened established customs and traditions, as well as their ignorance of the political, economic and social contexts that implicitly defined gender relations of their host communities. Even worse was the blatant dismissal and disrespect of host communities' cultures as inferior and the neglect of the internal dynamics of communities which they seek to transform positively.

To ameliorate this less than savoury side of this relationship, volunteer sending organizations must do better to prepare volunteers for their engagements with cultures of difference. IDVs can be better prepared by studying the geographical, political, socio-economic, and cultural contexts of their placement communities before arriving. When they arrive, their placements can be structured to ensure that they are continually receptive to further experiential knowledge on the gender norms that they seek to transform. Understanding local communities' interpretations of GEWE and engaging with the scholarly and activist works of feminists in the region would help IDVs frame their contributions to best suit their cultural context. For instance, there is a significant and rich literature on African feminisms that embraces a more conservative approach to GEWE while retaining traditional African values (Nnaemeka 2004; Nkeala 2016). To further learn from local contexts, IDVs can also link with locally based women's rights organizations and feminist organizations who can help communicate cultural realities while also helping local partners interpret the programming activities of IDVs using local gender expertise. 
Paramount to helping IDVs' develop greater cultural competence and a respect for relativism, they must be encouraged to engage in critical reflexivity on the power differentials between them and their host organizations and communities. Taking these power differences into account, IDVs can be more sensitive and respectful of local norms before proposing what and how change to gender dynamics should occur. The creation of space for critical reflection is not only consistent with ethical practice, it also carries the capacity to increase the impact and value of IDVs' presence in their host organizations and communities. As a further benefit, a clear respect for local priorities would endow GEWE program beneficiaries with a greater sense of empowerment because GEWE initiatives would not appear arbitrarily alien but would effectively reflect their own perceptions and meanings of gender equality and women's empowerment. Taking these actions, IDVs can aspire to emulate the example of the following volunteer working in Tanzania:

She is a person that used to reflect before speaking; to ask before sharing [...] She was a person that was ready to learn in every moment so, it was very difficult for her to [do or say] something that may result to conflict because she was very conscious to understand the values, the community situation, before she speaks, before she acts, before she shares anything... (see the paper in this collection by Tiessen, Laursen, Lough, with Mirza)

The importance of cultural sensitivity for the effective delivery of GEWE programming cannot be overemphasized. After all, IDVs' expertise and innovative ideas would serve no use if they were rejected outright by the communities they seek to help.

Findings from this collection also emphasize that the length of IDVs' placements is crucial to successful cultural immersion. If IDVs are to reasonably understand the cultural contexts and contribute meaningfully to gender equality and empowerment, significant time must be allowed for IDVs to first learn about the culture and context before acting or speaking on gender issues in the host communities where they are placed. These benefits of cultural immersion would not be feasible with short-term placements of less than three months; thus, longer placements are preferred. When local trainings are paired with cultural immersion of a sufficient duration, many of the potential problems evident in these papers could be avoided. Furthermore, these measures would help ensure strong rapport and trust between IDVs and their hosts.

Perhaps the most important theme, with clear implications for practice, is the importance of reciprocal learning and intercultural exchange between IDVs and Global South partner organizations. Much emphasis has been placed on the need for IDVs to be cognizant of cultural specificities of their host communities. However, partner organizations can also benefit from understanding the contexts that 
frame IDVs' feminist values. When Northern IDVs and Southern partners come together with minds open, ready, and prepared to learn, they will discover mutual understanding and genuine shared insights.

Although research that informed this collection was gathered in the South as an evaluation of North-South volunteer initiatives, Global South partners would also benefit from exchange opportunities to travel to the Global North. Transnational exchanges characterized by mutual cultural immersions from South to North can aid critical reflections on the distinct cultural practices in the Global North. This would allow Southern sojourners to critically appraise how their gendered relations intersect and diverge along lines of power and cultural expectations.

\section{Bibliography}

Nkealah, Naomi (2016): (West) African feminisms and their challenges. In: Journal of Literary Studies, vol. 32, no. 2, pp. 61-74, https://doi.org/10.1080/02564718.2016.1198156

Nnaemeka, Obioma (2004): Nego-feminism: Theorizing, practicing, and pruning Africa's way. In: Signs: Journal of Women in Culture and Society, vol. 29, no. 2, pp. 357-385, https:// doi.org/10.1086/378553.

Parpart, Jane L. (2014): Exploring the transformative potential of gender mainstreaming in international development institutions. In: Journal of International Development, vol. 26, no. 3, pp. 382-395, https://doi.org/10.1002/jid.2948. 